PREPARED FOR THE U.S. DEPARTMENT OF ENERGY, UNDER CONTRACT DE-AC02-76CH03073

PPPL-3620

PPPL-3620

UC-70

Stiff Temperature Profiles in JT-60U ELMy H-mode Plasmas

by

D.R. Mikkelsen, H. Shirai, H. Urano, T. Takizuka, Y. Kamada,

T. Hatae, Y. Koide, N. Asakura, T. Fujita, T. Fukuda, S. Ide,

A. Isayama, Y. Kawano, O. Naito, and Y. Sakamoto

October 2001

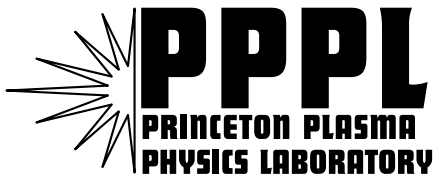

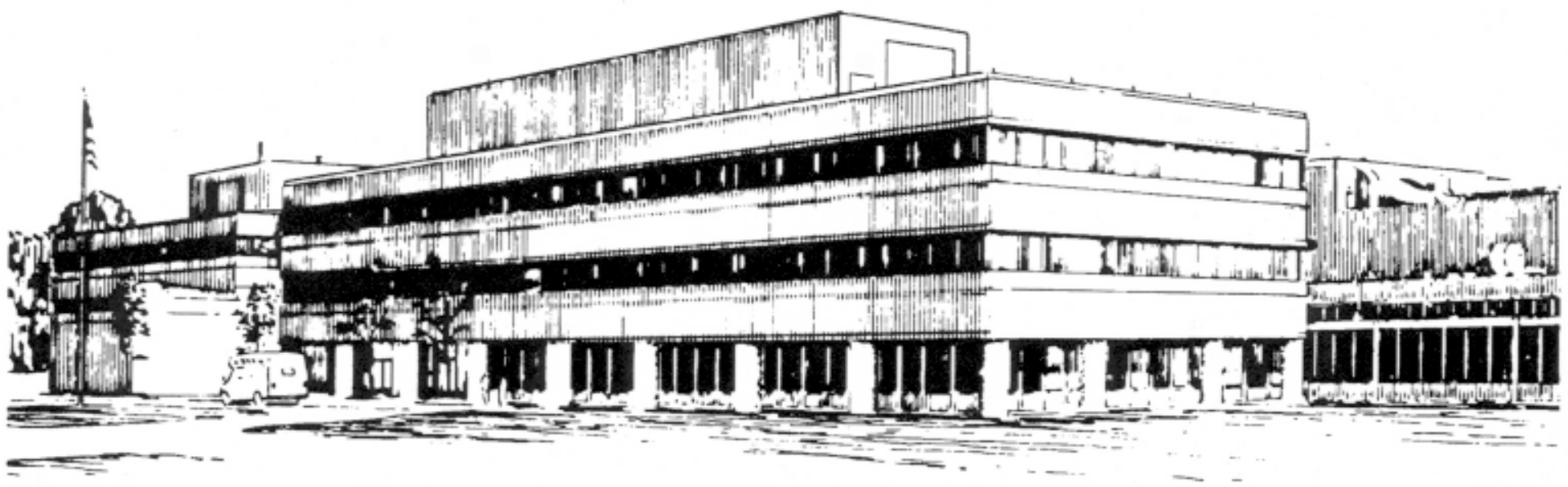

PRINCETON PLASMA PHYSICS LABORATORY PRINCETON UNIVERSITY, PRINCETON, NEW JERSEY 


\section{PPPL Reports Disclaimer}

This report was prepared as an account of work sponsored by an agency of the United States Government. Neither the United States Government nor any agency thereof, nor any of their employees, makes any warranty, express or implied, or assumes any legal liability or responsibility for the accuracy, completeness, or usefulness of any information, apparatus, product, or process disclosed, or represents that its use would not infringe privately owned rights. Reference herein to any specific commercial product, process, or service by trade name, trademark, manufacturer, or otherwise, does not necessarily constitute or imply its endorsement, recommendation, or favoring by the United States Government or any agency thereof. The views and opinions of authors expressed herein do not necessarily state or reflect those of the United States Government or any agency thereof.

\section{Availability}

This report is posted on the U.S. Department of Energy's Princeton Plasma Physics Laboratory Publications and Reports web site in FiscaL Year 2002. The home page for PPPL Reports and Publications is: http://www.pppl.gov/pub_report/

DOE and DOE Contractors can obtain copies of this report from:

U.S. Department of Energy

Office of Scientific and Technical Information

DOE Technical Information Services (DTIS)

P.O. Box 62

Oak Ridge, TN 37831

Telephone: (865) 576-8401

Fax: (865) 576-5728

Email: reports@adonis.osti.gov

This report is available to the general public from:

National Technical Information Service

U.S. Department of Commerce

5285 Port Royal Road

Springfield, VA 22161

Telephone: 1-800-553-6847 or

(703) 605-6000

Fax: (703) 321-8547

Internet: http://www.ntis.gov/ordering.htm 


\title{
Stiff Temperature Profiles in JT-60U ELMy H-mode Plasmas
}

\author{
D. R. Mikkelsen, Princeton Plasmas Physics Laboratory, \\ H. Shirai, H. Urano†, T. Takizuka, Y. Kamada, T. Hatae, Y. Koide, N. Asakura, \\ T. Fujita, T. Fukuda, S. Ide, A. Isayama, Y. Kawano, O. Naito, Y. Sakamoto, \\ JAERI Naka Fusion Research Establishment \\ $\dagger$ Hokkaido University
}

\begin{abstract}
The 'stiffness' of thermal transport in ELMy H-modes is examined in a series of carefully chosen JT-60U plasmas, and measured temperatures are compared with the predictions of several transport models. A heating power scan with constant $\mathrm{T}_{\text {ped }}$, a scan of pedestal temperature, $\mathrm{T}_{\text {ped }}$, with constant heating power, and an on-axis/off-axis heating comparison are presented. In the power scan a $45 \%$ increase in heating (and a $12 \%$ density rise) produces an approximately fixed core temperature profile in a group of five plasmas with the same pedestal temperature. With fixed heating power, we find that a $30-$ $40 \%$ increase in $\mathrm{T}_{\text {ped }}$ is associated with similar increases in core temperature. Heating in the deep core is varied by employing different groups of neutral beams that deposit their power near the magnetic axis and farther from the axis. In these plasmas on-axis heating produces slightly more peaked temperature profiles although they have $60 \%$ more heating power inside $\mathrm{r}=\mathrm{a} / 2$. Transport models are tested by solving the power balance equations to predict temperatures, which are then compared to the measurements. Predictions of the RLWB and IFS/PPPL models generally agree with the measured temperatures outside $\mathrm{r} \sim 0.3 \mathrm{a}$, but the Multimode model uniformly predicts temperatures that are too high except in the central region. Tests based on these discharges are not able to discriminate between the transport models of varying stiffness, so we conclude that larger changes are needed in the $\mathrm{P}_{\text {heat }}$ and $\mathrm{T}_{\text {ped }}$ scans
\end{abstract}




\section{Introduction}

Performance predictions for ITER (and other next-step tokamaks) depend strongly on the 'stiffness' of the assumed transport model [1,2]. This has motivated studies of transport stiffness in present tokamaks, and the development of experimental tests that might discriminate between transport models of differing stiffness. We describe JT-60U ELMy H-mode plasmas that exhibit profile stiffness, and we test the ability of several transport models to account for the observed linkage between core and peripheral temperatures $[3,4]$.

Temperature profile stiffness and more global indications of core-pedestal linkage have also been observed in T-10 [5], ASDEX-U [6, 7, 8, 9, 10, 11, 12], Alcator C-mod [13, 14], Tore Supra $[15,16]$ and FTU $[17,18,19]$. Both electron and ion temperatures have shown stiffness, but most of the evidence is based on electron temperatures - including the first observation in T-10 that the shape of the electron temperature profile is independent of the radial location of electron cyclotron heating [5]. Additional references are available in a recent review of electron thermal transport in tokamaks [20].

In the absence of internal transport barriers (which lead to very un-stiff profiles), neutral beam heated ELMy H-mode discharges in ASDEX-U have rather uniform temperature profile shapes with strong core-edge coupling $[6,7,8,11,12]$. The measured ion and electron temperatures are usually indistinguishable in typical high-density $\mathrm{H}$ mode discharges in ASDEX-U, and at low density the ion temperature profile remains stiff but the electron temperature profile is not stiff $[8,11,12]$. Variations in the neutral beam heating profile peakedness do not markedly alter the temperature profile shape [8]. Localized heating by ECH can change the electron temperature profile shape deep in the core, but the confinement region remains resilient $[9,10,11]$.

The ion and electron temperatures are tightly coupled in Alcator C-Mod so both are presumed to share the tight core-edge correlation seen in the electron temperature profile throughout a variety of L- and H-modes regimes $[13,14]$. The electron temperature profile shape changes very little in power scans with central heating in Tore Supra [15, 16]. FTU reports that the electron temperature profile is little changed when the location of off-axis electron cyclotron heating is varied [17, 19], and that peaked temperature profiles are observed in some plasmas with small (or even negative) net electron heating near the center [18].

Stiff profiles are not necessarily universal, however, as JET reports that varying the heating profile with on-axis vs. off-axis ICRF heating produces a significant change in the temperature profiles of $\mathrm{H}$-mode plasmas (although L-mode plasmas are notably less susceptible to changes), and core-edge coupling is not generally seen in JET H-mode plasmas [21]. However, stiff ion temperature profiles in JET have been reported in neutral beam heated plasmas with relatively high gas fueling rates [22].

Tests of transport models find that several models - of varying stiffness - are equivalently successful in representing a wide range of tokamak plasmas $[1,2]$. In 
particular, several transport models dominated by trapped electron modes (TEM) and ion temperature gradient (ITG) modes have reproduced the core-edge coupling seen in steady state ASDEX-U discharges [12]. In these simulations the ion temperature gradient is close to the theoretical critical gradient. More focused tests of transport stiffness based on transient phenomena provide additional insights, but clear discrimination between transport models has been difficult to achieve [23, 24]. Recently, however, the Weiland model [25] very successfully matched modulated ECH experiments in ASDEX-U [26, 27]. The measured (and predicted) electron temperature gradient is well above the critical gradient for trapped electron modes, as it is in Tore Supra [15, 16]; thus the electron temperature profiles are not very stiff [20].

In Section 2 we present evidence for stiff temperature profiles in ELMy H-mode plasmas in JT-60U. Pedestal temperature and heating power are independently varied, and we observe a strong correlation between core and peripheral temperatures; this correlation is largely independent of the heating power. The ion channel provides the strongest evidence for stiff transport in these discharges, but electron transport may also be stiff.

In Section 3 transport models are tested by solving electron and ion power balance equations to make temperature predictions for the discharges described in Section 2 . The IFS/PPPL [28] and RLWB [29] models' predictions are generally within $\sim 15 \%$ of the measured ion and electron temperatures in the 'confinement region', $0.3<\mathrm{r} / \mathrm{a}<0.7$, but the Multimode [23] model's ion temperature predictions are uniformly too high except in the central region. Nevertheless, predictions of all three tested models qualitatively resemble the observed linkage between core and peripheral temperatures.

\section{Experimental Results}

Our selection of plasmas is motivated by the defining characteristic of stiffness: large changes in conducted power are accommodated by relatively small changes in the temperature gradient. In 'critical gradient' theories there is no anomalous transport unless a critical temperature gradient [29] or temperature gradient scale length [28] is exceeded. Just beyond the stability threshold the conducted power is proportional to $\left(\nabla T-\nabla T_{\text {crit }}\right)$ or $\left(R / L_{T}-R / L_{T, \text { crit }}\right)$, respectively, and large fractional changes in conducted power can be caused by relatively small changes in $\nabla T$ or $L_{T}$ near threshold. If the proportionality constant is large enough then $\nabla T$ or $L_{T}$ will remain near their critical values.

In the case of a plasma that is near threshold all across the profile, and which is dominated by transport with a critical $L_{T}$, the shape of the temperature profile is determined by the profile of $L_{T, c r i t}$, and the core temperature is proportional to the edge temperature. If the transport is near threshold for a smaller region, $r<r_{\text {thr }}$, then $T_{\text {core }} \propto$ $\mathrm{T}\left(\mathrm{r}_{\text {thr }}\right)$. This fixed temperature profile shape and the proportionality of core and peripheral temperature is what is often meant by the expression 'stiff temperature profile', and may be caused by transport models with a critical $L_{T}$ (but not necessarily 
those with a critical $\nabla T$ ) which are near threshold over the region in which temperature proportionality holds. Transport models based on the ion temperature gradient (ITG) microinstability $[23,25,28]$ have the characteristics required to produce such 'stiff profiles'.

In idealized circumstances (and with constant heating power) a 'soft' transport model that is independent of temperature and its gradient will predict that core temperatures respond 'additively' to changes in the edge temperature, $\mathrm{T}_{\text {ped }}$, rather than proportionally as with a stiff model. With this kind of 'ideally soft' transport the temperature difference between two plasmas of a $\mathrm{T}_{\text {ped }}$ scan would be radially constant. With such a soft model the temperature rise from edge to core would be proportional to heating power, regardless of whether or not $\mathrm{T}_{\text {ped }}$ changed with heating power. If $\mathrm{T}_{\text {ped }}$ itself were also proportional to heating power, then even a soft transport model would predict that the entire profile would preserve its shape in a heating power scan. Thus, heating power scans can be used to differentiate between soft and stiff transport only if the power dependence of peripheral temperature can be minimized.

A group of plasmas with varying heating power but with fixed peripheral temperature (and fixed density, $I_{p}, B_{0}$, etc.) is well suited to test whether the energy transport is stiff. Conversely, a group of plasmas with varying peripheral temperature but with fixed heating power (and fixed density, $\mathrm{I}_{\mathrm{p}}, \mathrm{B}_{\mathrm{o}}$, etc.) is also appropriate. Finally, with on-axis vs. off-axis deposition the core heating can be varied while holding total heating constant, thus making it easier to maintain fixed peripheral temperature. JT-60U scans of the first two types are taken from pre-existing experiments [4], and the on-axis vs. off-axis heating experiment was carried out for this study.

The heating power scan (at fixed $\mathrm{T}_{\text {ped }}$ ) and the $\mathrm{T}_{\text {ped }}$ scan (at constant heating power) discussed below were selected from single day's run with ELMy H-mode discharges in JT-60U [4] with $\mathrm{I}_{\mathrm{p}}=1.8 \mathrm{MA}, \mathrm{B}_{\mathrm{O}}=3 \mathrm{~T}, \mathrm{R}_{\mathrm{O}}=3.2 \mathrm{~m}, \mathrm{a}=0.82-0.85 \mathrm{~m}, \kappa=1.5, \delta=0.16, \mathrm{q}_{95}=3$, $\bar{n}_{e}=2.5-3.1 \times 10^{19} \mathrm{~m}^{-3}, \mathrm{Z}_{\mathrm{eff}} \sim 2.6$, and $\mathrm{P}_{\mathrm{abs}}=6-10 \mathrm{MW}$. These plasmas have relatively low densities because that is where a wide range of $\mathrm{T}_{\text {ped }}$ is observed; with increasing density the type I ELM boundary dramatically reduces the $\mathrm{T}_{\text {ped }}$ range. The low power case in Figure 1 - it is also the high $\mathrm{T}_{\text {ped }}$ case in Fig. 2 - has a higher value of the confinement factor $\mathrm{H}$ than the comparison plasmas, which are good examples of typical energy confinement in JT-60U.

In the heating power scan, $\mathrm{T}_{\text {ped }}$ is apparently held fixed by ELM activity, which occurs more frequently as the power rises. This edge temperature clamping, together with the observed core-pedestal coupling, leads to strong power degradation of energy confinement once the type I ELM boundary has been reached [4]. In other discharges additional heating produces higher density and more stored energy, but for this study we have selected plasmas with a density close to that of the lower power plasmas. 
The plasmas with different $\mathrm{T}_{\text {ped }}$ at fixed Pheat, $\mathrm{I}_{\mathrm{p}}, \mathrm{B}_{\text {tor }}$, etc., occurred in different phases of the same JT-60U shot. They exhibit different types of ELM activity (type I and III), but this difference is likely a symptom of, not a cause of, the $\mathrm{T}_{\mathrm{ped}}$ difference. The plasma with lower $T_{\text {ped }}$ has a temperature lower than is typical for Type I ELMs. $T_{\text {ped }}$ is not correlated with $\mathrm{P}_{\text {rad }}$ or $\mathrm{Z}_{\text {eff }}$ but the different $\mathrm{T}_{\text {ped }}$ may be caused by the history of the edge conditions in the discharge. The case with lower density early in the heating phase is hotter then, and in the early heating phase both cases have grassy ELMS. Later, as the densities become similar the temperature difference diminishes, and different types of ELMs appear but the formerly low density case remains hotter than the initially denser plasma. Near the times of interest the $\mathrm{D}_{\alpha}$ intensity is $20-30 \%$ lower in the hotter plasma, and the difference was even greater about $0.5 \mathrm{sec}$ before the times of interest. This implies that charge exchange losses are lower in the hotter plasma, which could influence the temperatures near the edge, but not in the core where the neutral density is greatly attenuated. It is common to encounter variable edge conditions - and variable core confinement - in tokamaks, so other well matched pairs of discharges may be awaiting discovery. The correlation between core and edge confinement calls for further study to understand the factors that control $\mathrm{T}_{\text {ped }}$.

Each discharge contained a sequence of power steps from 5.5 to $10.5 \mathrm{MW}$ and a slow density ramp. The ion temperatures have been averaged for as long as possible (70 to 130 msec) in approximately steady conditions; beam faults and planned changes in beam power were the most common limiting events. The sawtooth phase is not synchronized and the averaging time is often shorter than a typical sawtooth period so it is advisable to ignore the sawtooth mixing region, $r<0.25 \mathrm{a}$, when comparing the measured temperature profiles to each other or to predicted profiles. The selection criteria were that the peripheral density and ion temperature match to within $\sim 10 \%$ to form the power scan, and that the density and heating power match to within $\sim 10 \%$ for $\mathrm{T}_{\text {ped }}$ scan.

In the power scan the ion temperature profiles show very little change at any radius (Figure 1a); the electron temperatures also exhibit no consistent power dependence but their measurement uncertainty and scatter are larger. In spite of a $45 \%$ increase in heating power both $\mathrm{T}_{\mathrm{e}}$ and $\mathrm{T}_{\mathrm{i}}$ are approximately constant, to $\leq 10 \%$. The density is correlated with power, causing $\bar{n}_{e}$ to rise $12 \%$ (Fig. 1b). The temperatures for the highest and lowest power discharges are shown in Fig. 1c and 1d; the measurement uncertainty of the ion temperature is smaller than the symbol size in the outer half of the plasma. The smooth curves in Fig. 1c are least squares fits of the form $T_{i}=A_{1}+A_{2} r^{2}+A_{3} r^{3}+A_{4} r^{4}$. Any systematic change in either temperature profile is not larger than the measurement uncertainties. The measured neutron rate rises with heating power slightly faster than linearly, so there is no evidence for enhanced fast ion losses (and a consequent reduction in heating) at higher power that might explain the constancy of the temperatures. The measured $\mathrm{P}_{\text {rad }}$ is $\sim 0.3 \mathrm{P}_{\text {heat }}$ for all power levels.

The measured temperatures from a $\mathrm{T}_{\text {ped }}$ scan are shown in Fig. $2 \mathrm{a}$ and $2 \mathrm{~b}$, and the electron density is shown in Fig. 2c. In idealized circumstances discussed above, very 'stiff' transport would produce a fixed temperature profile shape, with core temperatures 
proportional to $\mathrm{T}_{\text {ped. }}$ The constancy of the measured temperature profile shapes can be judged from the temperature ratios, $\mathrm{T}_{2} / \mathrm{T}_{1}$, shown in Figs. $2 \mathrm{~d}$, in which $\mathrm{T}_{1}$ corresponds to the lower $\mathrm{T}_{\text {ped }}$ discharge, and $\mathrm{T}_{2}$ corresponds to the discharge with higher $\mathrm{T}_{\text {ped }}$. The ion temperature ratio shown in Fig. $2 \mathrm{~d}$ decreases from the edge to the center, but it is approximately constant $(\sim 1.35$ to 1.4$)$ for $0.3<\mathrm{r} / \mathrm{a} \leq 0.85$. With 'soft' transport a $\mathrm{T}$ ped change at constant heating power produces a radially constant temperature difference, so the temperature difference $T_{2}-T_{1}$ is shown in Fig. 2e. A radially constant temperature difference is not consistent with the measured ion temperatures, but it is not ruled out for the electron temperatures. Thus, both of the idealized responses to the $\mathrm{T}_{\text {ped }}$ change are consistent with the measured electron temperature, which has much larger uncertainty.

Central heating power was varied in another group of H-mode discharges by alternately using neutral beams that pass near or avoid the magnetic axis (which was raised $0.25 \mathrm{~m}$ above the normal position near the midplane). Other discharge parameters are $\mathrm{I}_{\mathrm{p}}=1.5$ $\mathrm{MA}, \mathrm{B}_{\mathrm{O}}=3 \mathrm{~T}, \mathrm{R}_{\mathrm{O}}=3.4 \mathrm{~m}, \mathrm{a}=0.88 \mathrm{~m}, \kappa=1.4, \delta=0.3, \mathrm{q}_{95}=4.3, \bar{n}_{e}=2.6 \times 10^{19} \mathrm{~m}^{-3}$, $\mathrm{Z}_{\mathrm{eff}} \sim 2-2.5$, and $\mathrm{P}_{\mathrm{abs}}=4.2-5.5 \mathrm{MW}$. The measured temperatures and densities of one pair of plasmas in Fig. 3a-c show rather minor differences in spite of the much greater change in core heating shown in Fig. 3d. In another pair of plasmas in this series, the on-axis case has the higher peripheral $T_{i}$, and the $T_{i}$ profile is slightly more peaked with on-axis heating to a degree similar to that shown in Fig. 3a (where the on-axis case has the lower peripheral $\mathrm{T}_{\mathrm{i}}$ ). The larger uncertainties for the measured $\mathrm{T}_{\mathrm{e}}$ make it difficult to be certain of any change in the electron temperature profile shape. Faster central reheat with on-axis heating does cause slightly larger ion temperature differences in the early part of the sawtooth cycle, but profiles late in a sawtooth cycle are shown for comparison with the steady state predictions of the next section.

Fast ion losses caused by toroidal field ripple are included in the calculation of neutral beam heating profiles by TOPICS [30]/OFMC [31]. While the total power is approximately constant, the volume integrated ion heating is higher with on-axis heating, and the total electron heating is lower because a larger fraction of the beam power is deposited in the higher $\mathrm{T}_{\mathrm{e}}$ part of the plasma. Plasmas with on-axis heating have $60 \%$ more total heating power inside a/2 (Fig. 3d), which is supported by the measured DD neutron rate increase of $50 \%$. The $90 \%$ higher ion heating inside a/2 causes a much smaller increase in the core ion temperature (Fig. 3a). In the heating power and $\mathrm{T}_{\text {ped }}$ scans discussed above the heating profile shape changes very little.

\section{Transport Model Predictions}

Transport models are tested by solving the steady state electron and ion power balance equations to make temperature predictions for the discharges described above. The predictions are compared with the measured temperatures, and pairs of predictions are also compared with each other (e.g., high and low $\mathrm{T}_{\text {ped }}$ ). The tested transport models are the RLWB [29], the IFS/PPPL [28], and the Multimode [23] models. An ad hoc model with $\chi=C / n_{e}$ was used as a foil for the others; it represents a 'soft' thermal diffusivity 
with no dependence on temperatures or their gradients. The value of $\mathrm{C}$ was chosen separately for each scan, but it has the same value for both discharges within a scan.

The RLWB model has a critical $\nabla T_{e}$ (in both the electron and ion thermal diffusivities) so it can be expected to have a type of stiff electron transport (fractional change of conducted power $>>$ fractional change of $\nabla T_{e}$ near marginal stability), but this type of model does not ideally lead to a fixed temperature profile shape and proportionality between core and peripheral temperatures. The measured electron temperatures in the discharges discussed in Section 2 are consistent with a fixed $\nabla T_{e}$ in each pair of discharges: in the $\mathrm{T}_{\mathrm{ped}}$ scan the measured $\nabla T_{e}$ is approximately unchanged for $0.4 \mathrm{a}<\mathrm{r}$ $<0.7 \mathrm{a}$ (Fig. $2 \mathrm{~b}$ and see $2 \mathrm{e}$ ), and there is no clear change in the $\mathrm{T}_{\mathrm{e}}$ profile in either the heating power scan or the variation in central heating. This model is also distinctive in that it has Bohm-like scaling of the ion diffusivity and gyro-Bohm scaling of the electron diffusivity.

Both the IFS/PPPL and the Multimode models obey gyro-Bohm scaling, and both incorporate ion temperature gradient (ITG) mode transport with a critical $L_{T i}$ (although the theoretical treatments are quite different). Additional transport mechanisms are included in the Multimode model, but these generally are important near the plasma edge and near the magnetic axis so the Multimode model is dominated by its ITG component in the region around $\mathrm{r} \sim \mathrm{a} / 2$. The Multimode model incorporates an elongation dependence in its ITG term, thereby reducing its stiffness (this elongation dependence is not in Weiland's original formulation of ITG transport, but is a feature of the Multimode model itself).

The neutral beam heating profile used in the temperature predictions is calculated by TOPICS [30]/OFMC [31], which includes fast ion losses caused by toroidal field ripple. The measured density and temperatures have been mapped into flux surface coordinates, and the magnetic equilibria use MSE data to constrain the q profile. The power balance equations are solved by an iterative algorithm that uses predicted temperatures and measured $n_{e}(r), Z_{\text {eff, }} q(r)$, etc., to evaluate the transport model diffusivities, the convected power, and the electron-ion temperature equilibration power. Measured temperatures are used for the outer boundary condition at $\mathrm{r} / \mathrm{a}=0.82$ (the outermost position with reliably measured $\mathrm{T}_{\mathrm{i}}$ ).

Discrete sawteeth were not modeled, but a diffusivity of $1 \mathrm{~m}^{2} / \mathrm{sec}$ was added to each model inside $r=a / 5$ in order to approximately simulate the effects of sawtooth mixing. The size of the mixing region is estimated to $b e \leq \mathrm{a} / 4$ from the soft $\mathrm{X}$-ray sawtooth inversion radius. Temperature predictions for larger radii are not affected by more than $\sim 1 \%$, but predictions inside this region are not indicative of what the transport models alone would predict, so the models should not be judged by the predictions in the mixing region.

Predictions for discharges with the highest and lowest heating power with fixed $\mathrm{T}_{\mathrm{ped}}$ are shown in Figs. 4 and 5, respectively. The RLWB and IFS/PPPL models' predictions are typically within $15 \%$ of the measured ion temperatures outside $r=0.3 \mathrm{a}$, but the 
Multimode model's ion temperature predictions are too high except inside $\mathrm{r}=0.3 \mathrm{a}$. Note, however, that all three models' predictions are roughly as insensitive to the heating power change as the measured temperatures are, but the ad hoc model with $\chi=\mathrm{C} / \mathrm{n}_{\mathrm{e}}$ predicts a much larger temperature change. Both the Multimode and IFS-PPPL models' predictions are within the error bars of the measured electron temperature, but the RLWB is a bit lower near $\mathrm{r}=0.3 \mathrm{a}$. Again, the ad hoc model predicts a significantly larger change in the electron temperature when the heating power is varied.

A 'soft' transport model which is independent of temperature and its gradient will predict that core temperatures rise above $\mathrm{T}_{\text {ped }}$ by an amount which is proportional to the heating power. As shown in Fig. $6 \mathrm{a}$ and $6 \mathrm{~b}$, the predictions with $\chi=\mathrm{C} / \mathrm{n}_{\mathrm{e}}$ illustrate the expected linear scaling of $\left(\mathrm{T}-\mathrm{T}_{\mathrm{ped}}\right)$ with heating power (discharge 1 has the lower heating power, discharge 2 the higher). The electron temperature ratio in Fig. $6 \mathrm{~b}$ for $\chi=\mathrm{C} / \mathrm{n}_{\mathrm{e}}$ is smaller that the ion ratio in Fig. 6a because the predicted higher $\mathrm{T}_{\mathrm{e}}$ of the higher power case causes $\mathrm{Q}_{\mathrm{ie}}$ to transfer a lower fraction of the heating power to the electrons than in the lower power case. The extremely large uncertainties in the experimental values for the ratio of $\left(\mathrm{T}-\mathrm{T}_{\text {ped }}\right)_{2} /\left(\mathrm{T}-\mathrm{T}_{\text {ped }}\right)_{1}$ are caused by the low values of $\left(\mathrm{T}-\mathrm{T}_{\text {ped }}\right)$ in the periphery.

Figs. 5 and 7 show the measured and predicted temperatures for the $T_{\text {ped }}$ scan, with the higher and lower $\mathrm{T}_{\text {ped }}$, respectively. The RLWB and IFS/PPPL models' predictions are within $15 \%$ of the measured ion temperatures for $r>0.3 \mathrm{a}$, while the Multimode model's predictions are too high except near the center.

To better determine whether temperature profile shapes are preserved, the ratio of $\mathrm{T}_{2} / \mathrm{T}_{1}$ is shown in Fig. 8 (discharge 1 has the lower $\mathrm{T}_{\text {ped }}, 2$ has higher $\mathrm{T}_{\text {ped }}$ ). Both the RLWB and IFS-PPPL models' predictions preserve the shapes to the same extent that was observed in the experiment. Curiously, the Multimode predictions are as 'soft' as those of $\chi=C / n_{e}$. The ion temperature difference, $T_{2}-T_{1}$, shown in Fig. $8 \mathrm{c}$ also shows that only the IFS-PPPL model predicts much more than an 'additive' temperature response in the deep core. However, the larger measurement uncertainties of the relatively small values of the electron temperature difference (Fig. 8d) encompass all the models' predictions.

The electron temperatures are changed less that the ion temperatures, especially at the location of the boundary condition used in the predictions. This leads to relatively small changes in the predicted $\mathrm{T}_{\mathrm{e}}$, which are generally within $15 \%$ of the measured temperatures although the RLWB predictions are even lower near $\mathrm{r} \sim 0.3 \mathrm{a}$ (Fig. $5 \mathrm{~b}$ and $7 \mathrm{~b}$ ). None of the models' predicted ratio of $\mathrm{T}_{2} / \mathrm{T}_{1}$ falls outside the measured ratio (Fig. $8 \mathrm{~b}$ ).

The predictions of the on-axis vs. off-axis heating experiment (Fig. 9) are qualitatively similar to those described above. The Multimode $T_{i}$ predictions are too high, except near the center, and - in a departure from the previous cases - the predicted electron temperature is too high even near the center. The IFS/PPPL model's predicted $T_{i}$ change from off-axis to on-axis heating is smaller than the measured one, and its predicted $\mathrm{T}_{\mathrm{i}}$ is systematically too cold for $r<0.4 \mathrm{a}$. Otherwise, the RLWB and the IFS/PPPL model 
predictions are again near the measured temperatures outside the central region, and both models' predictions are near the measured electron temperatures. The ad hoc $\chi=\mathrm{C} / \mathrm{n}_{\mathrm{e}}$ model predicts the largest change in temperatures, followed by the RLWB model and the Multimode model, while the IFS/PPPL model is again the stiffest - even stiffer than the measured temperatures.

\section{Conclusions}

ELMy H-mode discharges in JT-60U exhibit stiff temperature profiles in the plasma core; this is particularly true for the ion temperatures, as predicted by theories of ion temperature gradient mode turbulence. In a power scan with fixed pedestal temperature, a pedestal temperature scan with fixed heating power, and a heating redistribution experiment, we observe that the core temperatures respond roughly proportionally to changes in the peripheral temperatures but are relatively insensitive to changes in heating

power. In the $T_{\text {ped }}$ scan the core ion temperature changes are larger than the changes in the periphery, and cannot be matched by a simple additive change in temperature across the profile. The larger ion temperature pedestal change provides the strongest evidence for stiff transport in these discharges, but the electron energy transport may also be stiff.

The peripheral temperatures are apparently limited in the heating power scan by type I ELM activity so it may be possible to carry out similar experiments in other tokamaks as well. It may also be possible to extend the range of $\mathrm{P}_{\text {heat }}$ scan in JT-60U to lower and higher powers, but maintaining a constant density could be difficult.

Three transport models have been tested by comparing their predicted temperature profiles to the measured temperatures. The Multimode model's predictions for ion temperature are too hot, but they scale as the measured temperatures do in the heating power scans and the $\mathrm{T}_{\text {ped }}$ scan. The RLWB and Multimode models' predictions are similar to the measured temperatures, and the predicted responses to heating and $\mathrm{T}_{\text {ped }}$ variations are much closer to the experimental results than are the predictions of an $a d$ hoc soft transport model. However, tests based on these discharges are not able to discriminate between the theoretically based transport models of varying stiffness, so we conclude that definitive tests require larger changes in the $\mathrm{P}_{\text {heat }}$ and $\mathrm{T}_{\text {ped }}$ scans.

[1] Mikkelsen, D. R., et al., "Tests of 1-D Transport Models, and their Predictions for ITER", in Fusion Energy (Proc. 17th Intl. Conf., Yokohama, 1998) CN-69/ITERP1/08.

[2] ITER Physics Basis, Chapter 2.8, Nucl. Fusion 39 (1999) 2215-2225.

[3] Asakura, N., et al., Plasma Phys. Control. Fusion, 39 (1997) 1295-1314.

[4] Urano, H., et al., to be published in Nuclear Fusion, 2001.

[5] Alikaev, V. V., et al., Sov. J. Plasma Phys. 14 (1988) 601.

[6] Suttrop, W., et al., Plasma Phys. Control. Fusion, 39 (1997) 2051-2066.

[7] Suttrop, W., et al., Plasma Phys. Control. Fusion, 42 (2000) A97-A102.

[8] Stober, J. et al., Plasma Phys. Control. Fusion, 42 (2000) A211-A216.

[9] Ryter, F., et al., Phys. Rev. Letters, 86 (2001) 2325.

[10] Ryter, F., et al., Phys. Rev. Letters, 86 (2001) 5498.

[11] Ryter, F., et al., Nuclear Fusion, 41 (2001) 537. 
[12] Tardini, G., et al., "Comparison of theory based transport models with ASDEX Upgrade data", to appear in Nuclear Fusion.

[13] Greenwald, M., et al., Nuclear Fusion 37 (1997) 793.

[14] Greenwald, M., et al., Plasma Phys. Control. Fusion, 40 (1998) 789-792.

[15] Hoang, G. T., et al., "Eelctron transport and improved confinement in Tore Supra", in Fusion Energy (Proc. 18th Intl. Conf., Sorrento, 2000) CN-77/EX6/1.

[16] Hoang, G. T., et al., Phys. Rev. Letters, 87 (2001) 125001.

[17] Sozzi, C. et al., "Energy confinement and sawtooth stabilization by ECRH at high electron density in FTU tokamak", in Fusion Energy (Proc. 18th Intl. Conf., Sorrento, 2000) CN-77/Exp5/13.

[18] Jacchi, A., et al., "Gradient length driven transport in EC heated FTU tokamak", $14^{\text {th }}$ Topical Conf. on Radio Frequency Power in Plasmas, May 2001, Oxnard.

[19] Cirant, S., et al, "Impact on MHD stability and energy confinement of localized ECRH in FTU tokamak", $14^{\text {th }}$ Topical Conf. on Radio Frequency Power in Plasmas, May 2001, Oxnard.

[20] Ryter, F., et al., "Experimental studies of electron transport", to appear in 2001 EPS issue of Plasma Phys. Control. Fusion.

[21] Christiansen, J. P., et al., "The plasma profile response to variations of the heating profile", 26th European Physical society Conference on Controlled Fusion and Plasma Physics, (Maastricht, The Netherlands, 1999) ECA Vol. 23J (1999) 209.

[22] Horton, L. D., et al., Plasma Phys. Control. Fusion, 41 (1999) B329.

[23] Kinsey, J. E. , Waltz, R. E., and DeBoo, J. C., Phys. Plasmas, 6 (1999) 1865-1871.

[24] Neu, R., et al., "Propagation of cold pulses and heat pulses in ASDEX-Upgrade", in Fusion Energy (Proc. 18th Intl. Conf., Sorrento, 2000) CN-77/EXP5/33.

[25] Froejdh, M., Christiansen, J. P., Strand, P., and Weiland, J., J. Plasma Phys. Control. Fusion 38, 326 (1996).

[26] Tardini, G. Peeters, A.G., Pereverezev, G.V., Ryter, F.. Stober, J., and the ASDEX Upgrade team, "Comparison of theory based transport models with ASDEX Upgrade data", 27th European Physical society Conference on Controlled Fusion and Plasma Physics, (Budapest, Hungary, 2000) ECA Vol. 24B (2000) 1148.

[27] Tardini, G., et al., "Theory based modeling of ASDEX Upgrade discharges with ECH modulation", submitted to Nuclear Fusion.

[28 ] Kotschenreuther, M., et al., Phys. Plasmas, 2 (1995) 2381.

[29] Rosenbluth, M. N., et al., in Fusion Energy (Proc. 15th Intl. Conf., Seville, 1994) 2, 517.

[30] Shirai H., et al., J. Phys. Soc. Japan, 64 (1995) 4209.

[31] Tani, K., Azumi, M., Kishimoto, H. , and Tamura, S., J. Phys. Soc. Japan, 50 (1981) 1726. 


\section{Figure Captions}

Fig. 1) a) Measured ion temperature at selected radii vs. total heating power. For the lowest power (solid line) and highest power (dashed line) plasmas: b) electron density profiles, c) ion temperature profiles, d) electron temperature profiles.

Fig. 2) Two plasmas with low (solid line) and high (dashed line) pedestal temperature: a) ion temperature profiles, b) electron temperature profiles, c) electron density profiles. Temperature ratios and differences for electrons (solid line) and ions (dashed line): $d$ ) the ratio (higher $\mathrm{T}_{\text {ped }}$ llower $\mathrm{T}_{\text {ped }}$ ), and e) difference (higher $\mathrm{T}_{\text {ped }}-$ lower $\mathrm{T}_{\text {ped }}$ ).

Fig. 3) Plasmas with on-axis (solid line) and off-axis (dashed line) heating: a) ion temperatures, b) electron temperatures, c) electron density, d) integrated electron + ion heating power.

Fig. 4) Measured and predicted temperatures for the plasma with higher heating power in Figure 1: a) ion temperatures, b) electron temperatures. Symbols with error bars are measured temperatures; curves are predictions for $\chi=C / n_{\mathrm{e}}$ (dot-dash line), Multimode model (solid line), Rebut-Lallia-Watkins-Boucher model (short-dash line), and IFS-PPPL model (long-dash line).

Fig. 5) Measured and predicted temperatures for the plasma with lower heating power in Figure 1 and high $\mathrm{T}_{\text {ped }}$ in Figure 2: a) ion temperatures, b) electron temperatures. Symbols with error bars are measured temperatures; curves are predictions for $\chi=C / n_{\mathrm{e}}$ (dot-dash line), Multimode model (solid line), Rebut-Lallia-Watkins-Boucher model (short-dash line), and IFS-PPPL model (long-dash line).

Fig. 6) Ratio of temperature off-set with respect to boundary condition for the power scan plasmas in Figure 1: a) ion temperature, and b) electron temperature. Symbols with error bars are derived from measured temperatures; curves are derived from predictions for $\chi=\mathrm{C} / \mathrm{n}_{\mathrm{e}}$ (dot-dash line), Multimode model (solid line), Rebut-Lallia-Watkins-Boucher model (short-dash line), and IFS-PPPL model (long-dash line).

Fig. 7) Measured and predicted temperatures for plasma with low $T_{\text {ped }}$ in Figure 2: a) ion temperatures, b) electron temperatures. Symbols with error bars are measured temperatures; curves are predictions for $\chi=\mathrm{C} / \mathrm{n}_{\mathrm{e}}$ (dot-dash line), Multimode model (solid line), Rebut-Lallia-Watkins-Boucher model (short-dash line), and IFS-PPPL model (longdash line).

Fig. 8) Ratio of temperatures for the $T_{\text {ped }}$ scan in Figure 2: a) ion temperature, and $b$ ) electron temperature, and temperature difference: c) ion temperature, and d) electron temperature. Symbols with error bars are derived from measured temperatures; curves are derived from predictions for $\chi=\mathrm{C} / \mathrm{n}_{\mathrm{e}}$ (dot-dash line), Multimode model (solid line), RebutLallia-Watkins-Boucher model (short-dash line), and IFS-PPPL model (long-dash line). 
Fig. 9) Measured and predicted temperature for plasma with off-axis heating in Figure 3:

a) ion temperature, b) electron temperature; and for on-axis heating: c) ion temperature, $d$ ) electron temperature. Symbols with error bars are measured temperatures; curves are predictions for $\chi=\mathrm{C} / \mathrm{n}_{\mathrm{e}}$ (dot-dash line), Multimode model (solid line), Rebut-LalliaWatkins-Boucher model (short-dash line), and IFS-PPPL model (long-dash line). 
Figure 1
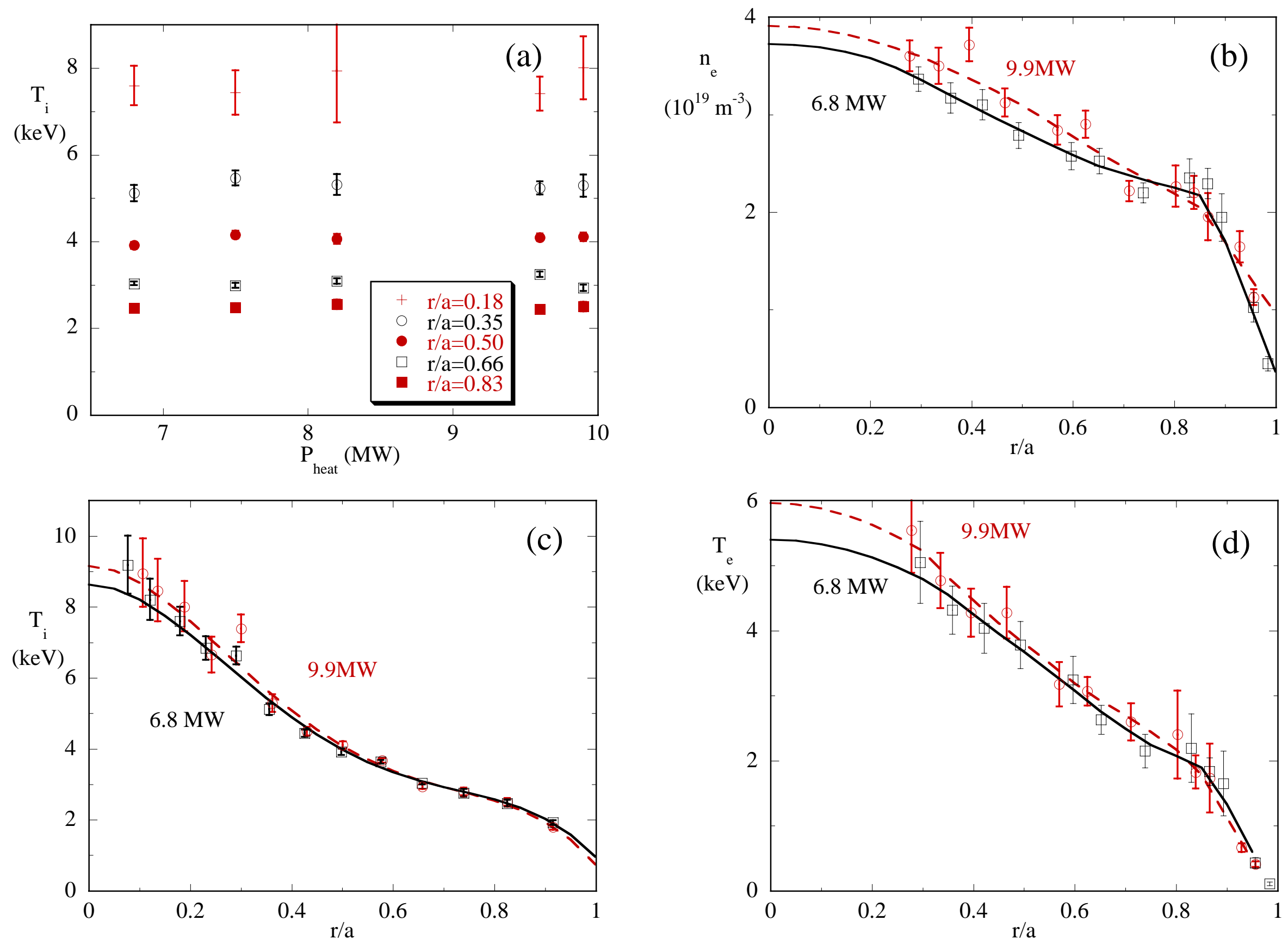

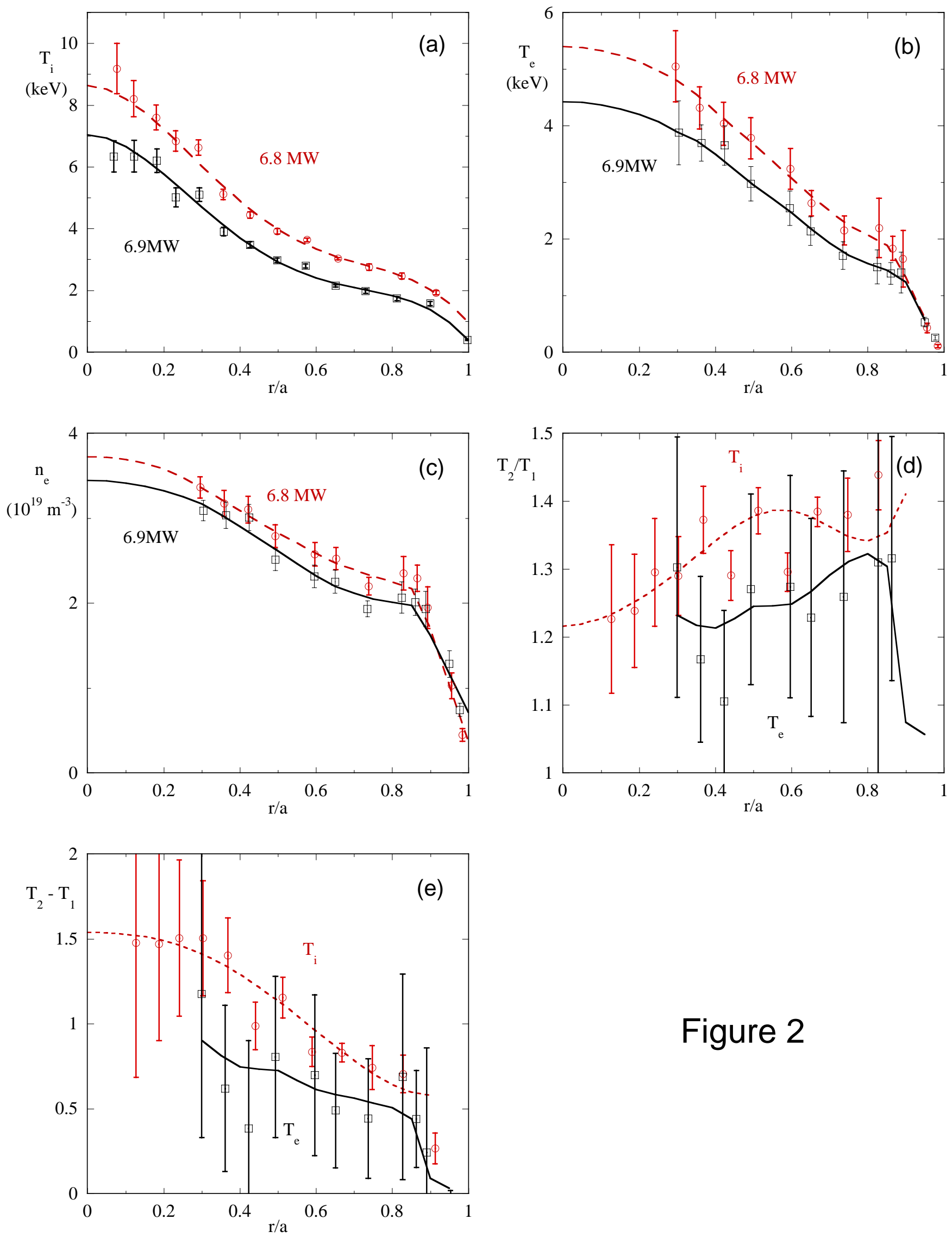

Figure 2 
Figure 3
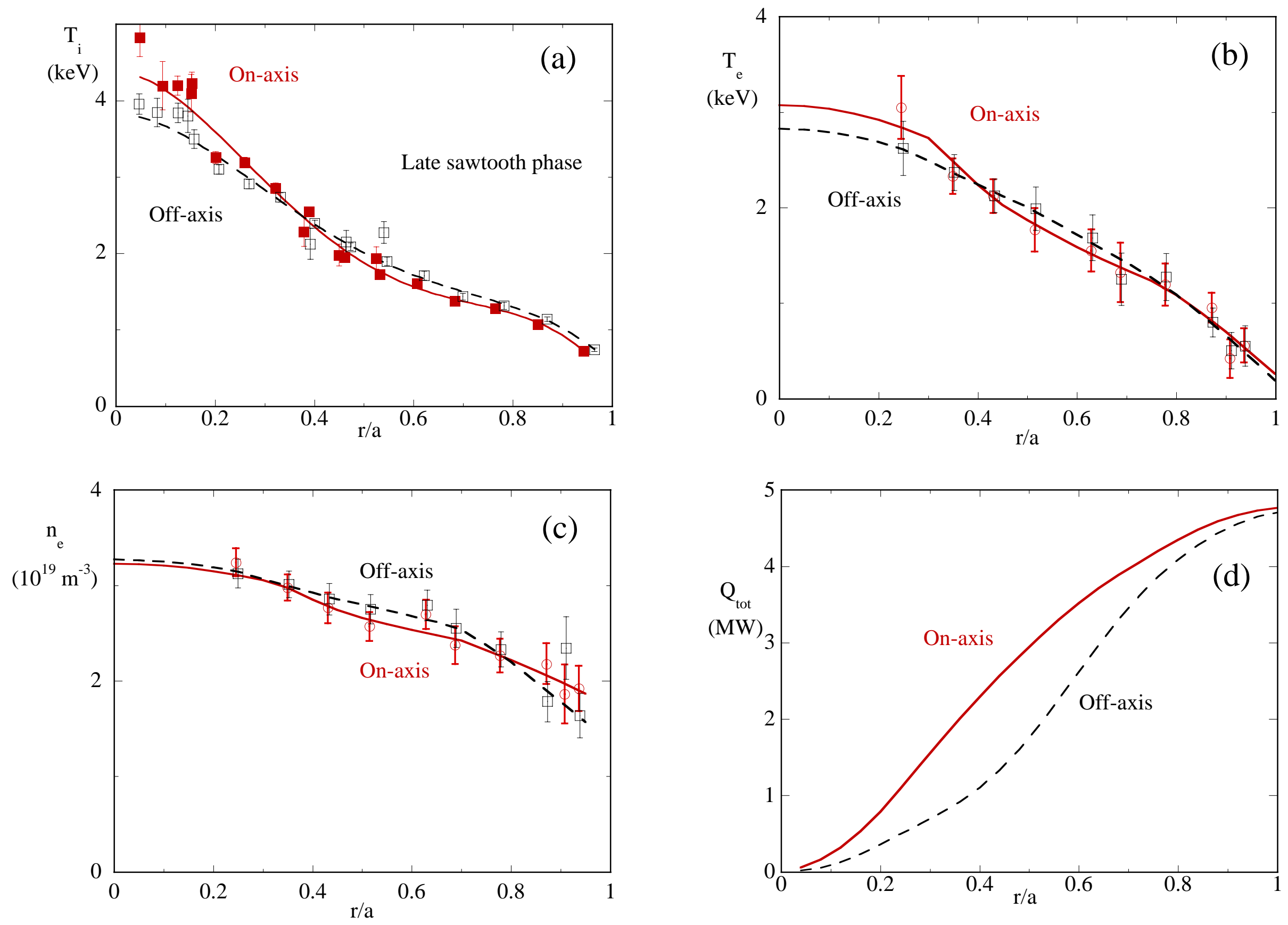
Figure 4
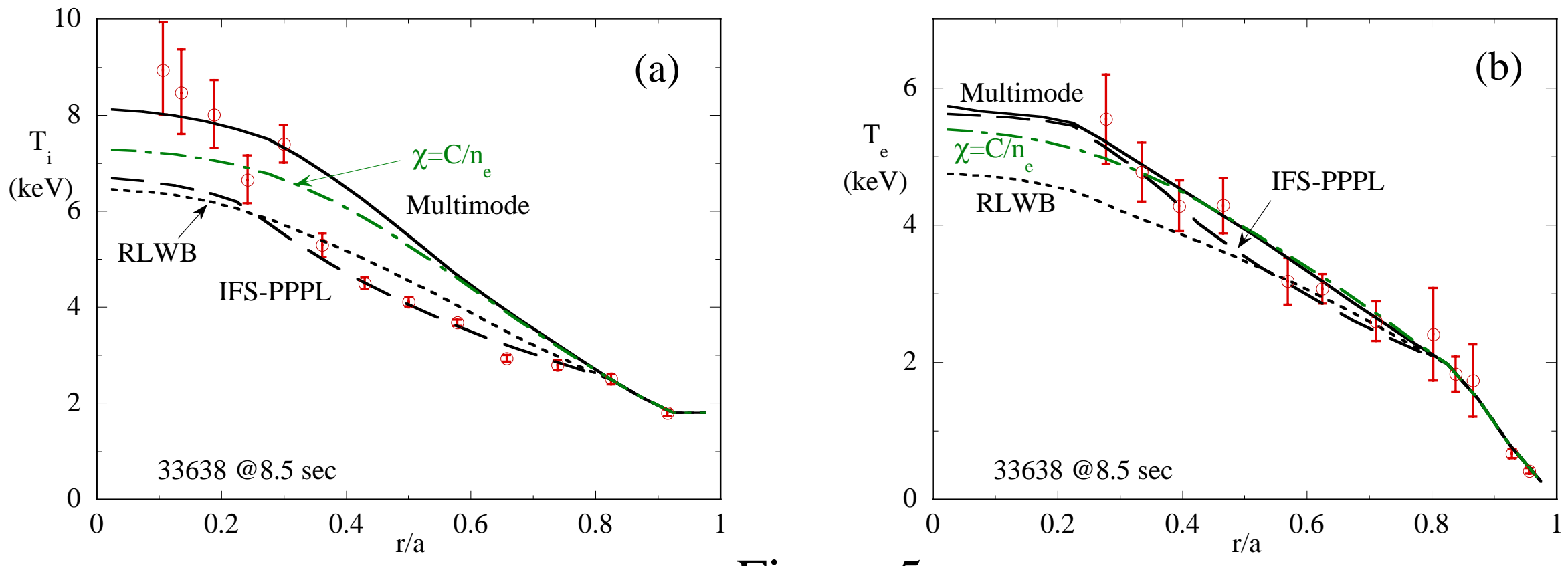

Figure 5
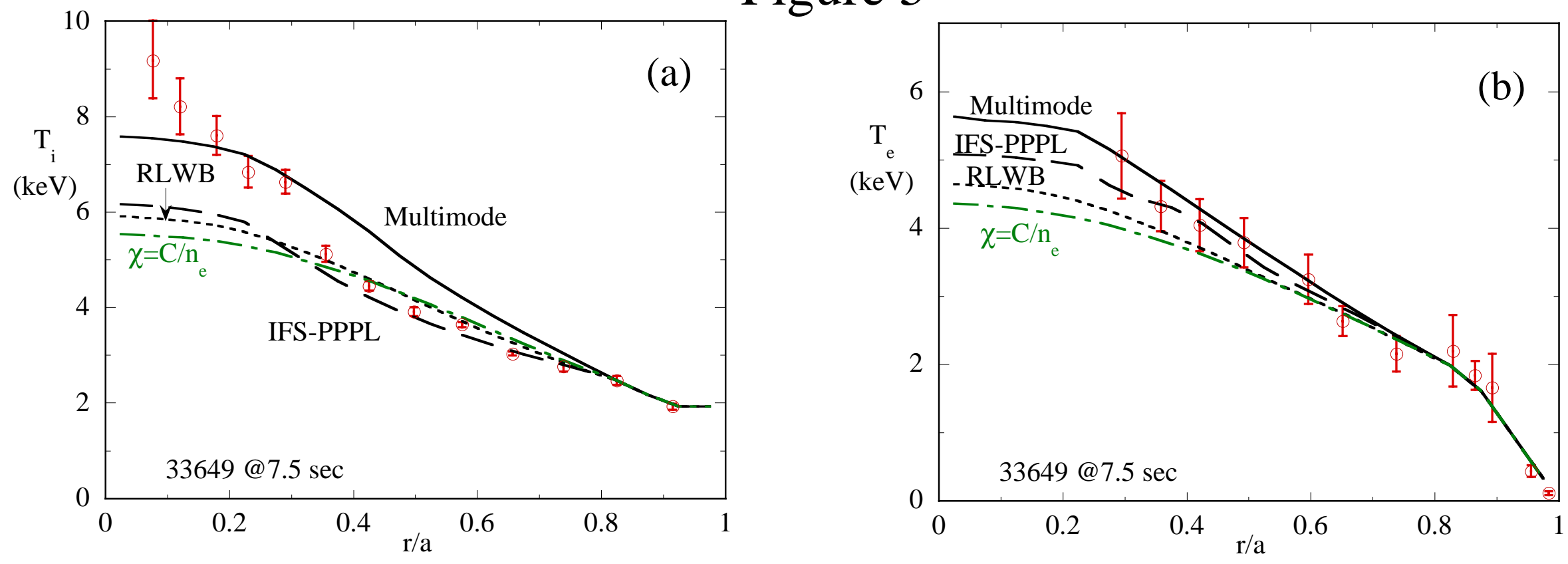
Figure 6
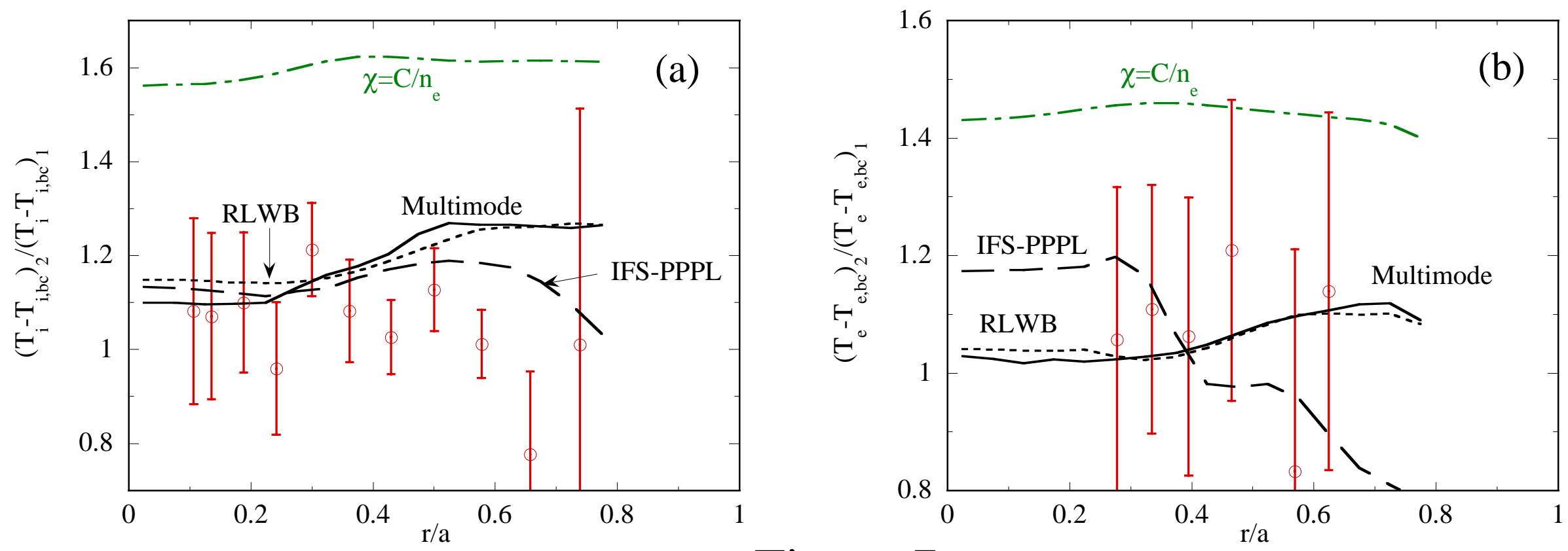

Figure 7
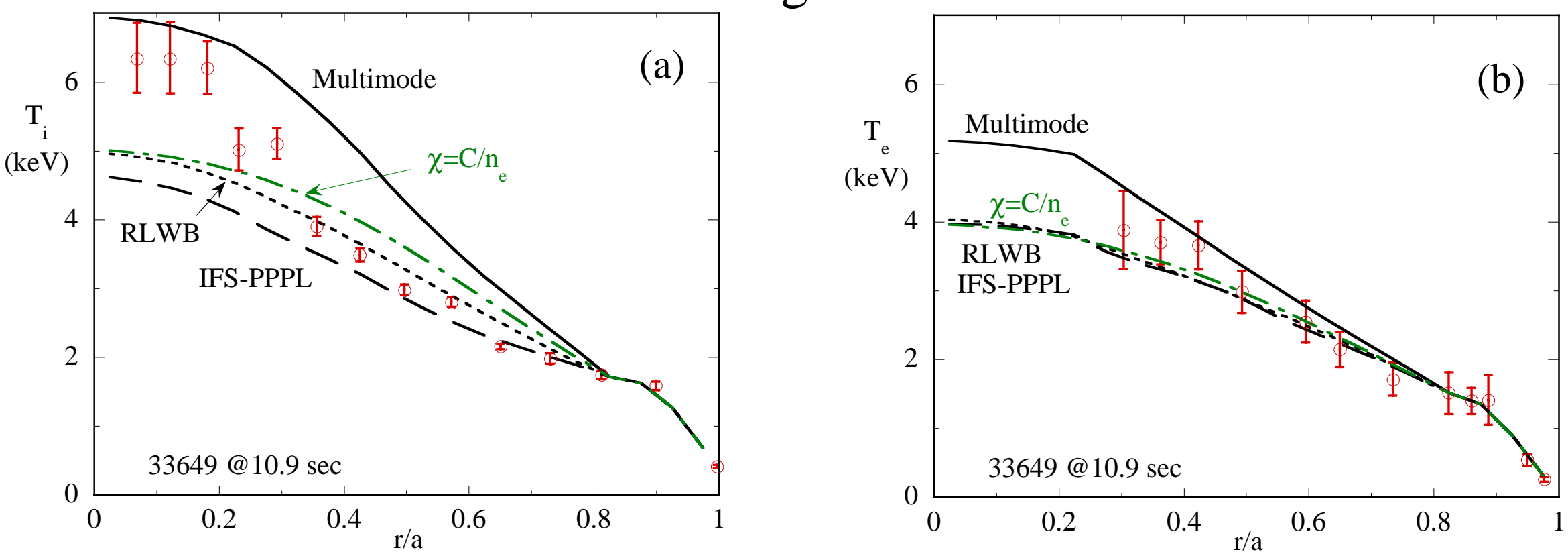
Figure 8
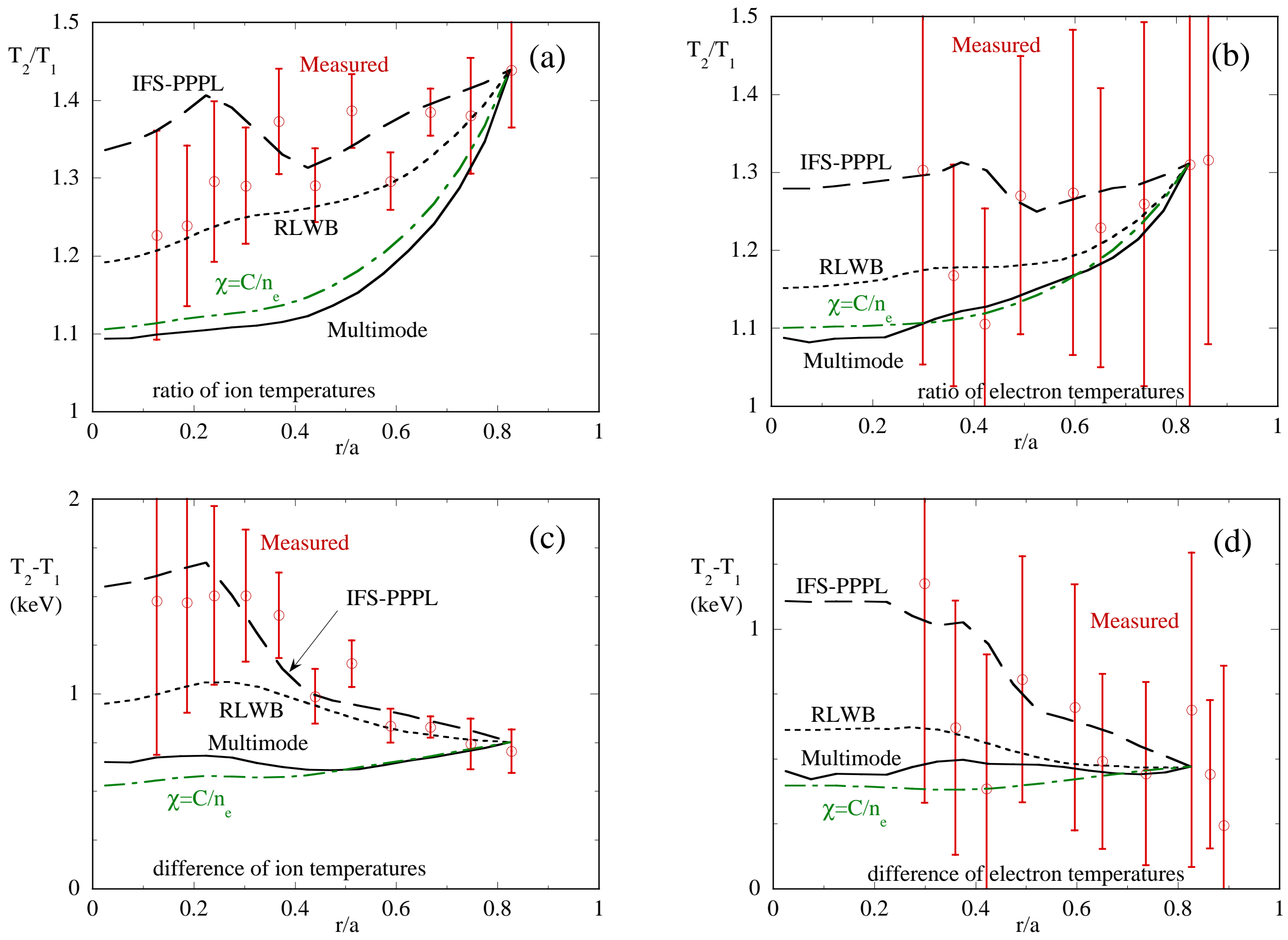
Figure 9
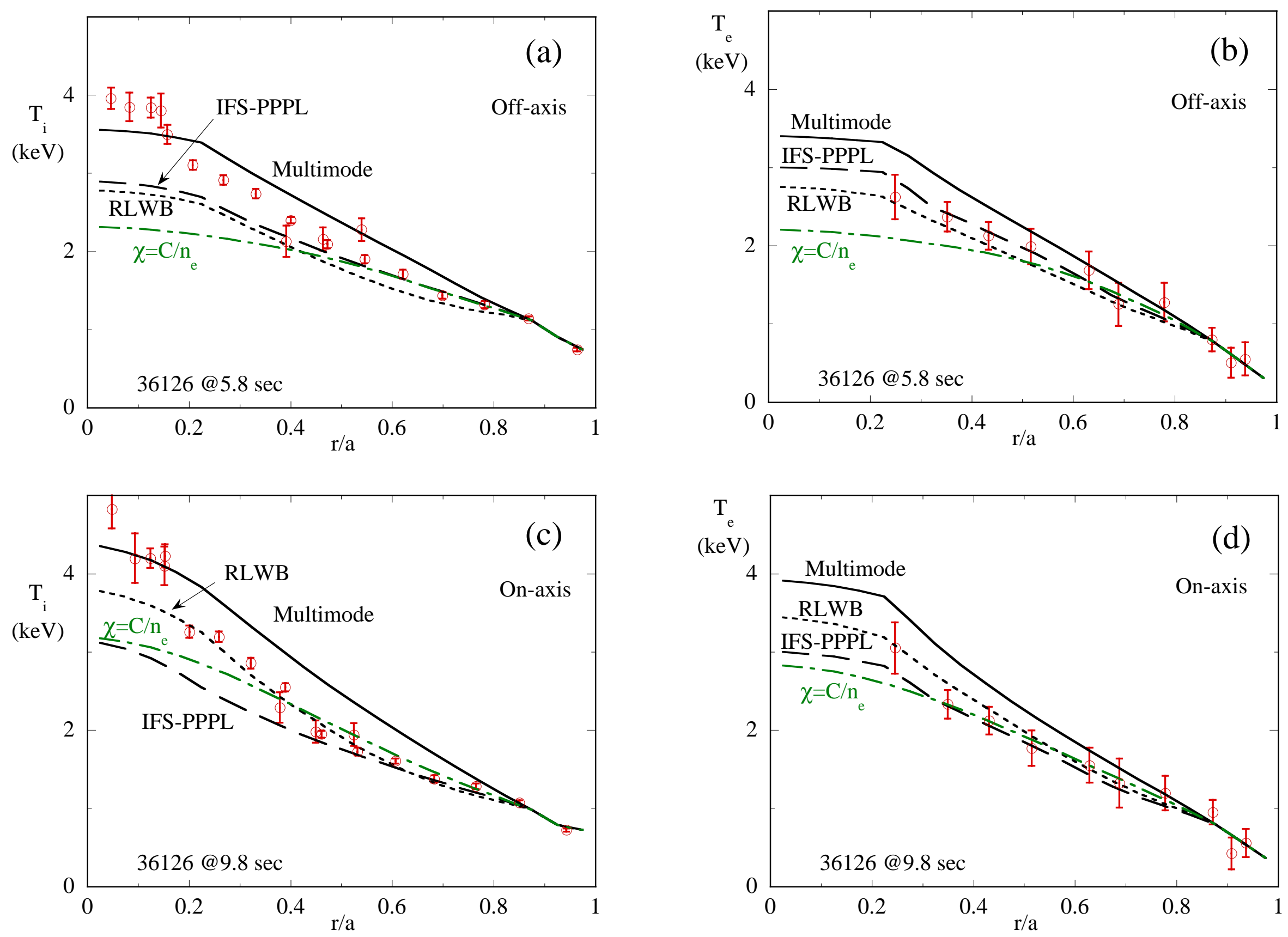


\section{External Distribution}

Plasma Research Laboratory, Australian National University, Australia

Professor I.R. J ones, Flinders University, Australia

Professor J oão Canalle, Instituto de Fisica DEQ/IF - UERJ , Brazil

Mr. Gerson O. Ludwig, Instituto Nacional de Pesquisas, Brazil

Dr. P.H. Sakanaka, Instituto Fisica, Brazil

The Librarian, Culham Laboratory, England

Library, R61, Rutherford Appleton Laboratory, England

Mrs. S.A. Hutchinson, JET Library, England

Professor M.N. Bussac, Ecole Polytechnique, France

Librarian, Max-Planck-Institut für Plasmaphysik, Germany

J olan Moldvai, Reports Library, MTA KFKI-ATKI, Hungary

Dr. P. Kaw, Institute for Plasma Research, India

Ms. P.J . Pathak, Librarian, Insitute for Plasma Research, India

Ms. Clelia De Palo, Associazione EURATOM-ENEA, I taly

Dr. G. Grosso, Instituto di Fisica del Plasma, Italy

Librarian, Naka Fusion Research Establishment, J AERI, J apan

Library, Plasma Physics Laboratory, Kyoto University, J apan

Research Information Center, National Institute for Fusion Science, J apan

Dr. O. Mitarai, Kyushu Tokai University, J apan

Library, Academia Sinica, Institute of Plasma Physics, People's Republic of China

Shih-Tung Tsai, Institute of Physics, Chinese Academy of Sciences, People's Republic of China

Dr. S. Mirnov, TRINITI, Troitsk, Russian Federation, Russia

Dr. V.S. Strelkov, Kurchatov Institute, Russian Federation, Russia

Professor Peter Lukac, Katedra Fyziky Plazmy MFF UK, Mlynska dolina F-2, Komenskeho Univerzita, SK-842 15 Bratislava, Slovakia

Dr. G.S. Lee, Korea Basic Science Institute, South Korea

Mr. Dennis Bruggink, Fusion Library, University of Wisconsin, USA

Institute for Plasma Research, University of Maryland, USA

Librarian, Fusion Energy Division, Oak Ridge National Laboratory, USA

Librarian, Institute of Fusion Studies, University of Texas, USA

Librarian, Magnetic Fusion Program, Lawrence Livermore National Laboratory, USA

Library, General Atomics, USA

Plasma Physics Group, Fusion Energy Research Program, University of California at San Diego, USA

Plasma Physics Library, Columbia University, USA

Alkesh Punjabi, Center for Fusion Research and Training, Hampton University, USA

Dr. W.M. Stacey, Fusion Research Center, Georgia Institute of Technology, USA

Dr. J ohn Willis, U.S. Department of Energy, Office of Fusion Energy Sciences, USA

Mr. Paul H. Wright, Indianapolis, Indiana, USA 
The Princeton Plasma Physics Laboratory is operated by Princeton University under contract with the U.S. Department of Energy.

\author{
Information Services \\ Princeton Plasma Physics Laboratory \\ P.O. Box 451 \\ Princeton, NJ 08543
}

Phone: 609-243-2750

Fax: 609-243-2751

e-mail: pppl_info@pppl.gov

Internet Address: http://www.pppl.gov 